Avicenna Journal of Nursing and Midwifery Care - ISSN 2676-5748

\title{
The Prevalence of Thyroid Disorders in Pregnant Women of Hamadan
}

\author{
Shiva Borzouei ${ }^{*}$, MohammadTaghi Goodarzi ${ }^{2}$, Mansooreh Biglari ${ }^{3}$, Fathollah Nazari $^{3}$, \\ Zahra Shivapour ${ }^{4}$ \\ 1. Assistant Professor, Department of Endocrinology, Faculty of Medicine, Hamadan University of Medical \\ Sciences, Hamadan, Iran \\ 2. Research Center for Molecular Medicine, Hamadan University of Medical Sciences, Hamadan, Iran \\ 3. General Physician, Hamadan University of Medical Sciences, Hamadan, Iran \\ 4. MSc in Epidemiology, Hamadan University of Medical Sciences, Hamadan, Iran
}

\begin{tabular}{lr}
\hline \multicolumn{2}{c}{ Article Info } \\
\hline & \\
Received: & $2018 / 07 / 26$ \\
Accepted: & $2018 / 10 / 10$ \\
Published Online: & $2019 / 03 / 22$
\end{tabular}

DOI: $10.30699 /$ ajnmc.27.1.11

Original Article

Use your device to scan and read the article online

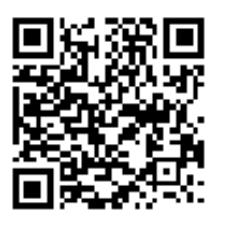

\section{Abstract}

Introduction: Different studies have demonstrated that maternal thyroid dysfunction during pregnancy is associated with adverse obstetrics and fetal outcomes. There is no international consensus regarding to use a guidelines for screening in high risk women. The aim of the present study was to determine prevalence of thyroid disorders in high and low risk pregnant women.

Methods: In a cross-sectional study during 2015-2016, from all pregnant women who had referred to Hamadan health center Lab for their first visit, a sample of 852 pregnant women were selected and assigned to low risk and high risk group. Thyroid tests and Anti TPO tests were carried out.

Results: Of 852 pregnant women, $26.5 \%$ had Subclinical hypothyroidism, 1.2\% had overt hypothyroidism, $0.5 \%$ had overt hyperthyroidism and $0.2 \%$ had subclinical hyperthyroidism. $25.6 \%$ were in the high risk group and $74.4 \%$ in the low risk group and $37.4 \%$ of high risk group and $25 \%$ of the low risk population had thyroid disorders $(P<0 / 001) .89 \%$ were Anti TPO negative and $11 \%$ were Anti TPO positive.

Conclusion: Hypothyroidism is common in pregnant women, and if screening is performed only in high-risk groups, $25 \%$ of pregnant women with subclinical and overt hypothyroidism will not be detected explicitly. In addition, to determine the exact frequency of thyroid disorders, we need to look for newer and more effective criteria.

Keywords: Pregnancy, Thyroid Disorders, Risk Factor
Corresponding Information
Shiva Borzouei, Assistant Professor, Department of Endocrinology, Faculty of Medicine, Hamadan University of Medical Sciences, Hamadan, Iran. Email: shivapoorzahra@yahoo.com

Copyright (C) 2019, This is an original open-access article distributed under the terms of the Creative Commons Attribution-noncommercial 4.0 International License which permits copy and redistribute of the material just in noncommercial usages with proper citation. 
مجلة مراقبت يرستارى و مامايى ابنسينا - شايا الكترونيك: UVY-DVA

مقاله يزوهشى

بررسى ميزان شيوع اختلالات تيروئيدى در خانمهاى باردار شهر همدان

شيوا برزويى ":، محمدتقى تودرزى"، منصوره بيعلرى"، فتحاله نظرى"، زهرا شيوايور"

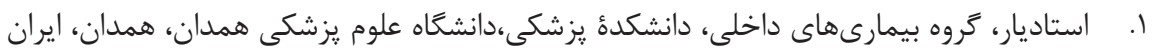

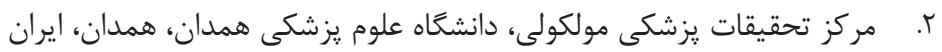

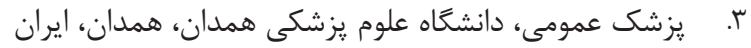

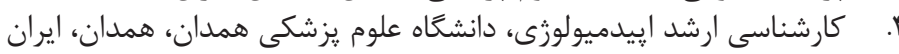

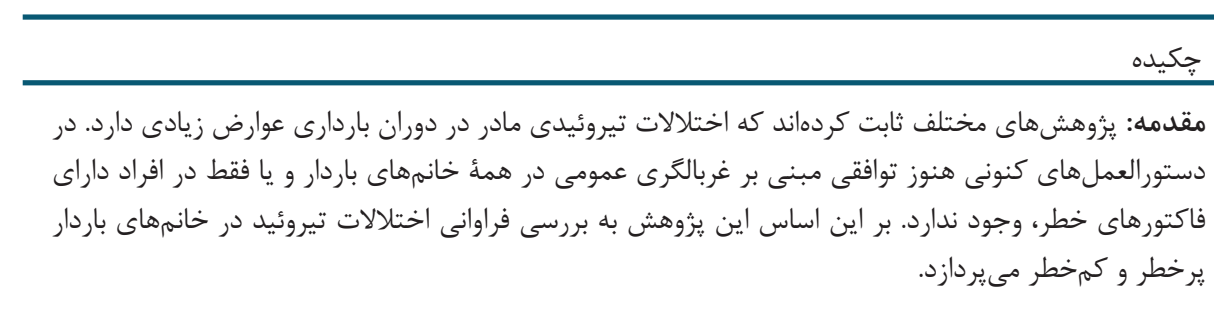

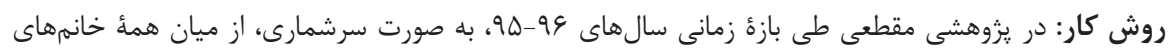

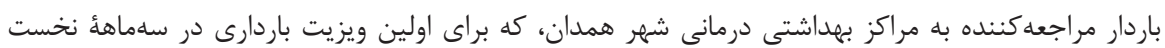

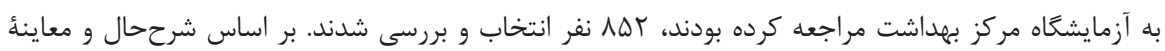

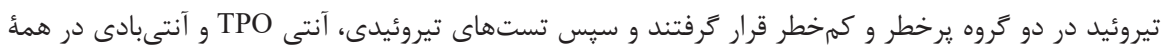

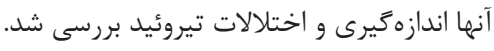

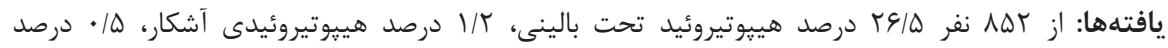

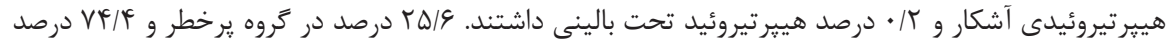

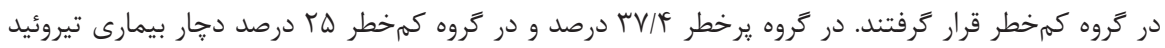

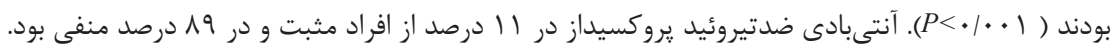

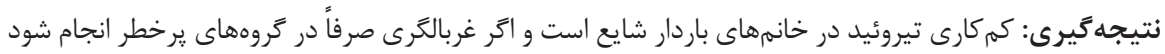

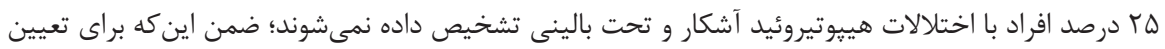

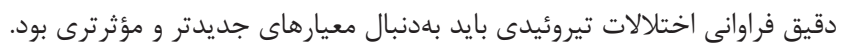

كليدوازهها: باردارى، اختلالات تيروئيدى، عامل خطر

$$
\begin{aligned}
& \text { تاريخ وصول: } \\
& \text { نويسندهُ مسئول: } \\
& \text { شيوا برزويى } \\
& \text { استاديار، كروه بيمارىهاى برويى } \\
& \text { داخلى، دانشكدة يزشكى، }
\end{aligned}
$$

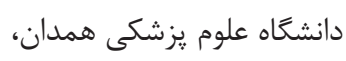

$$
\begin{aligned}
& \text { همدان، ايران } \\
& \text { يست الكترونيك: }
\end{aligned}
$$

shivapoorzahra@yahoo.com

$$
\text { مادر و جنين كاهى بسيار مشكل است [ه ، [F] }
$$

^ادرصد زنان باردار آنتىبادى تيروئيد يروكسيداز و

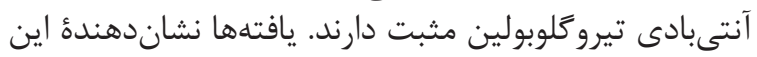

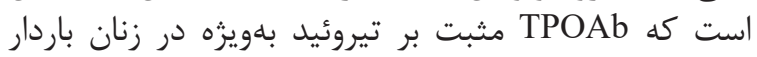

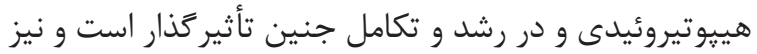

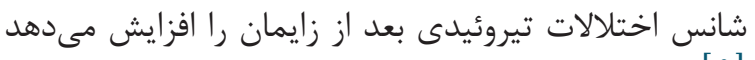

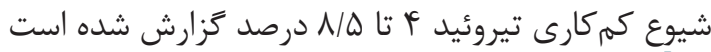

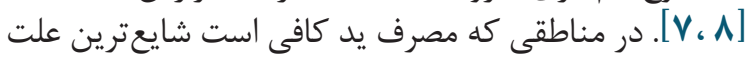

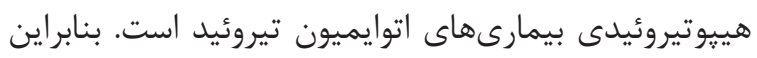

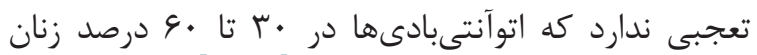

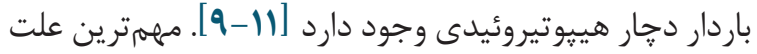

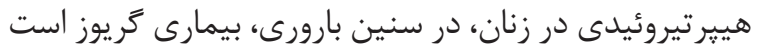

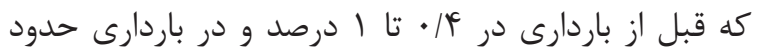

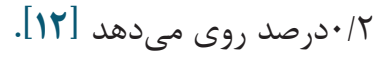

دورة

مقدمه

باردارى تأثير عمدهاى بر غده تيروئيد و عملكرد آن دارد.

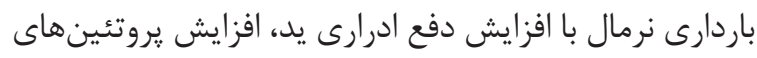

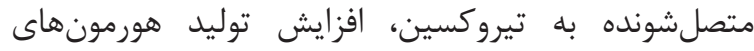

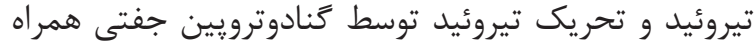

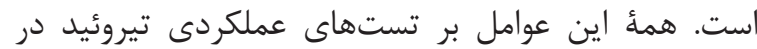

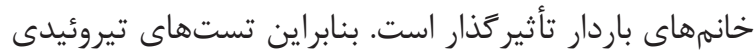

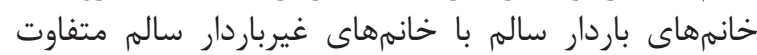

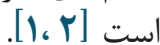

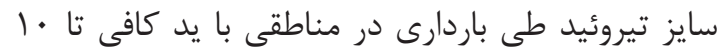

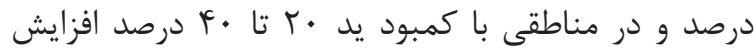

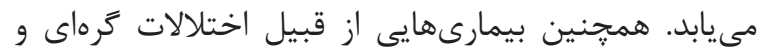

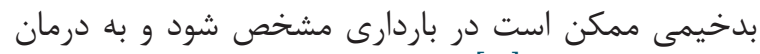

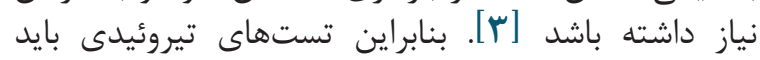

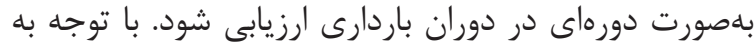

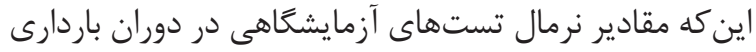

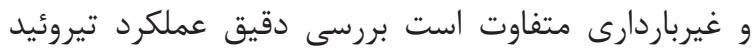


• سطح آنتىبادىAnti-TPO با استفاده از روش

ELISA

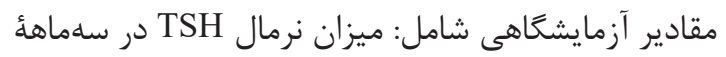

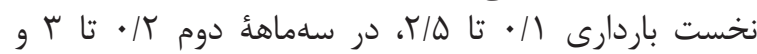

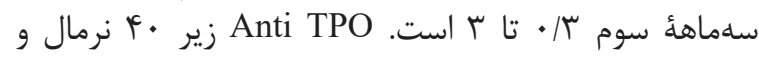

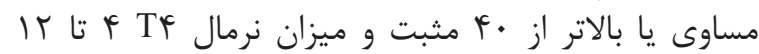

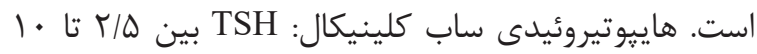

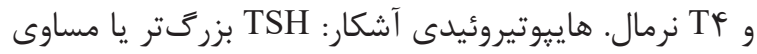

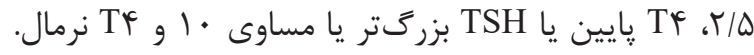

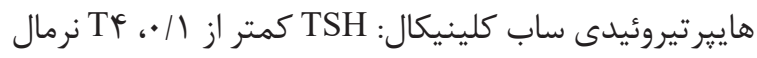

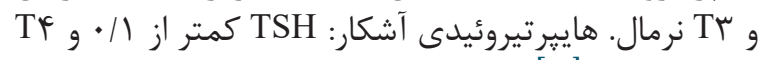

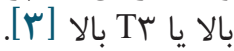

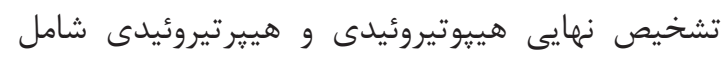

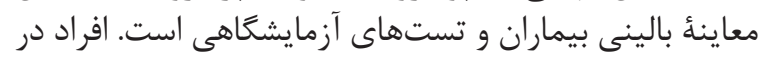

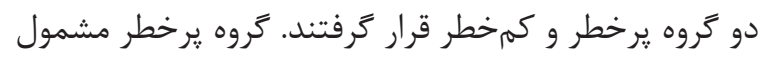

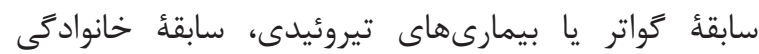

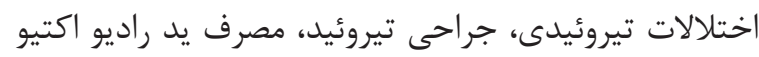

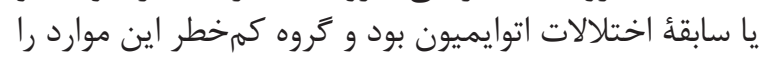

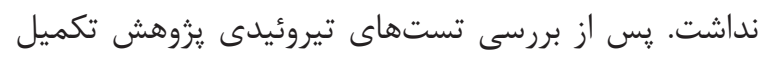

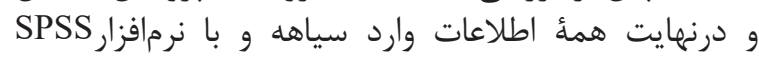

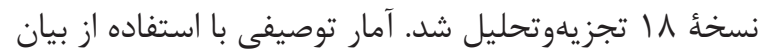

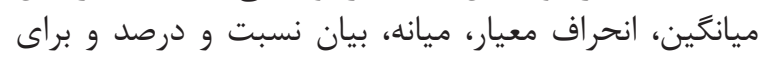
مقايسٔ فراوانى متغيرها از كائ اسكور استفاده شد.

\section{يافتهها}

ميانگَين سنى مادران باردار شركت كننده در يروهش

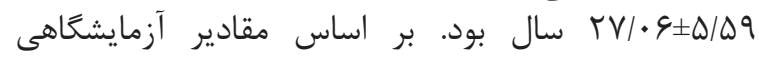

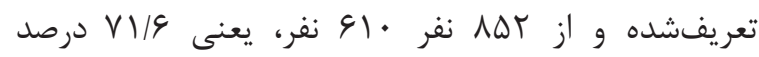

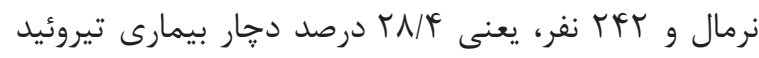

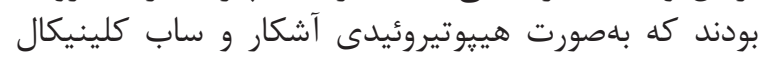

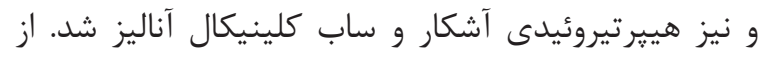

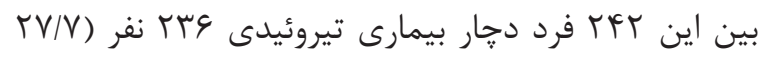

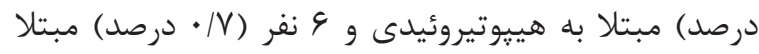

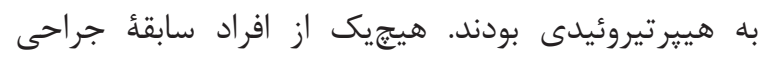

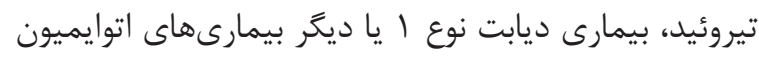

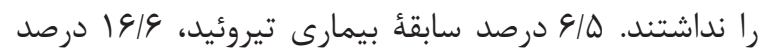

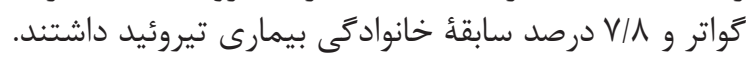
درصد زيركروههاى بيمارى تيروئيد در نمودار ا آمده

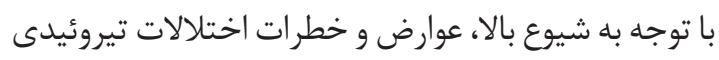

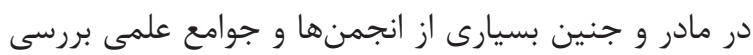

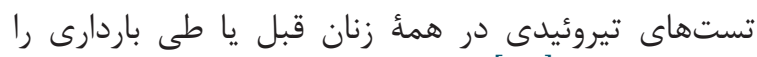

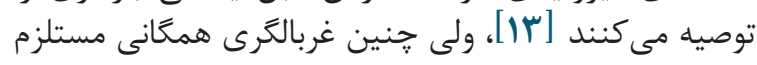

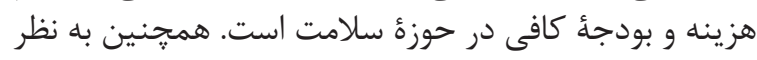

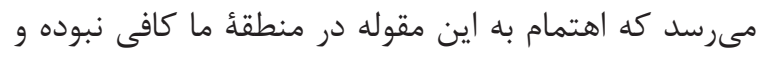

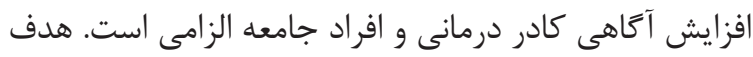

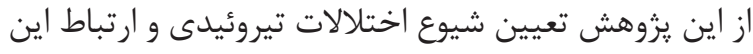

اختلال با عوامل خطر در زنان باردار شهر همدان است.

$$
\text { روش بررسى }
$$

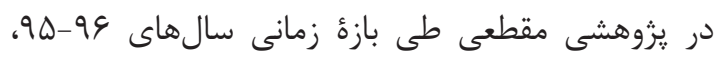

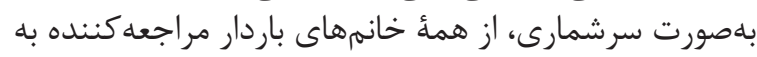

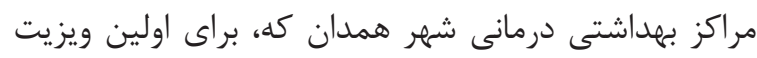

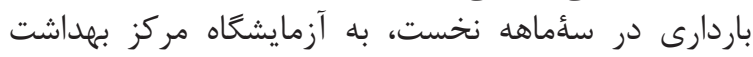
مراجعه كرده بودند

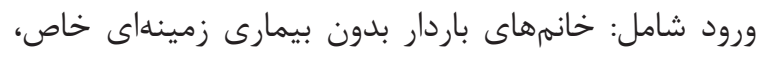

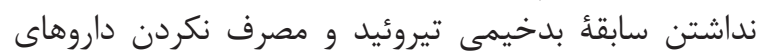

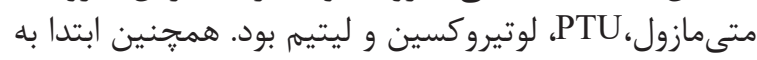

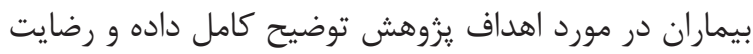

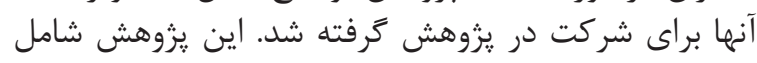

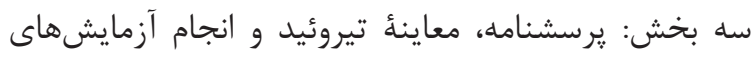
مرتبط بود.

1. يرسشنامه: شامل اطلاعات دموكر افيك (سن، كراويد،

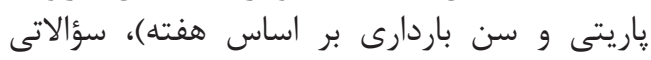

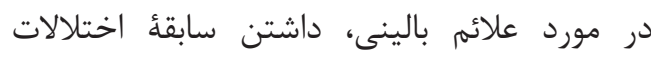

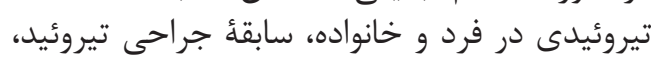

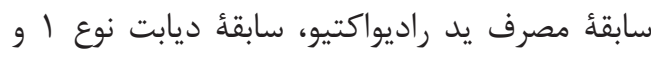
بيمارى هاى اتوايميون بود.

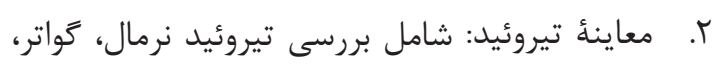

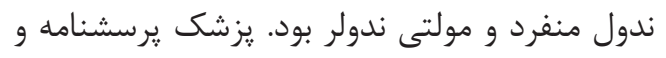
معاينٔ تيروئيد را تكميل مى مردى ندول

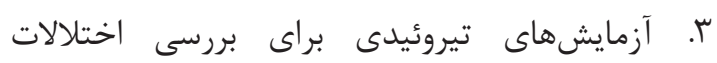

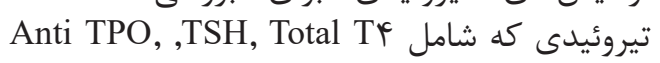
TrRu

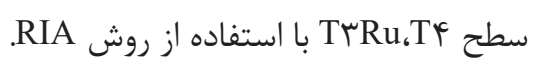

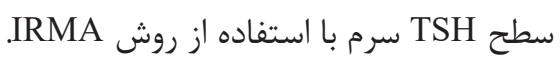

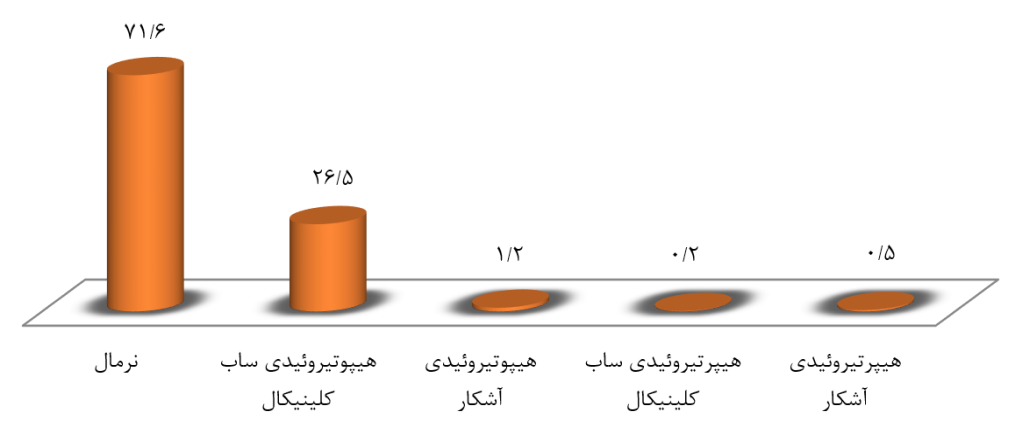

نمودار ا. درصد فراوانى افراد نرمال و بيمارى تيروئيد در شركت كنندكان 


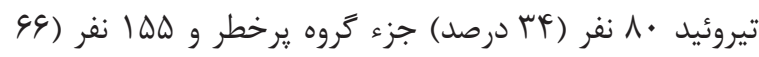

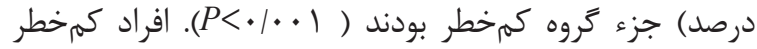

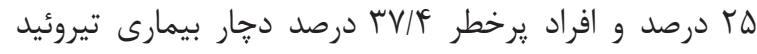

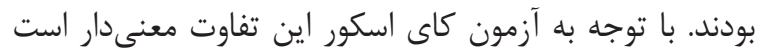
. $(P<\cdot 1 \cdot 1)$

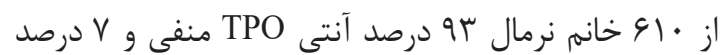
آنتى TPO مثبت، از

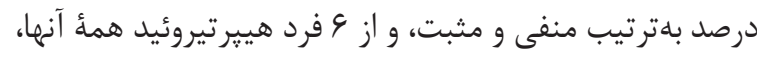

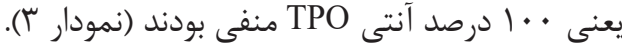

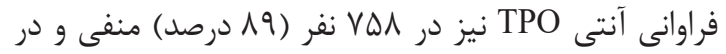

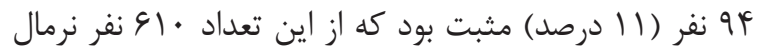

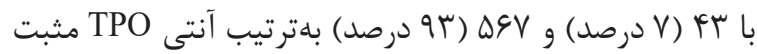

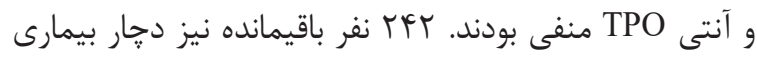

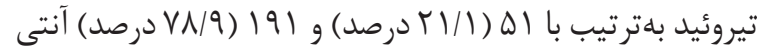

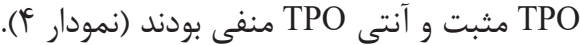

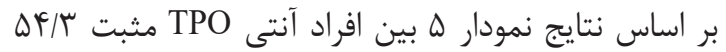

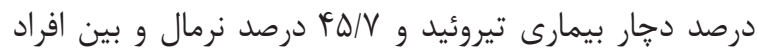

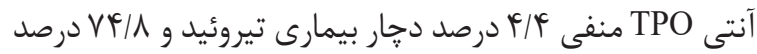

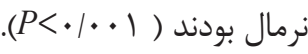

بر اساس وجود هريك از عوامل خطر بيمارى تيروئيد بيماران

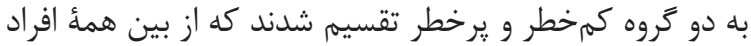

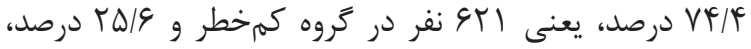

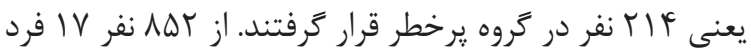

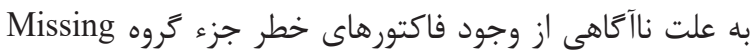

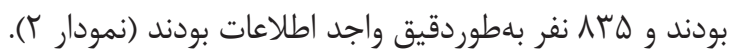

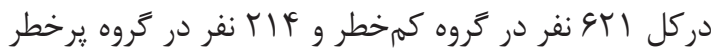

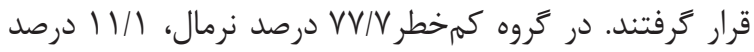
هييوتيروئيدى آشكار، 99// 9 درصد هيبيوتيروئيدى ساب كلينيكال

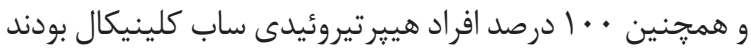

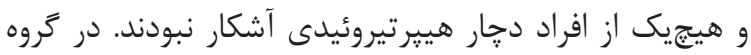

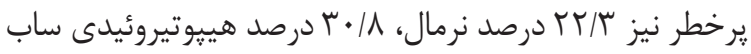

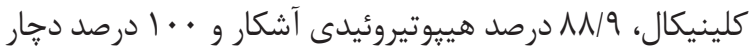

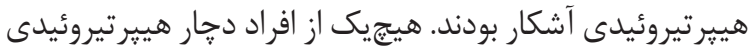

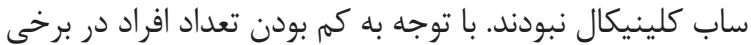

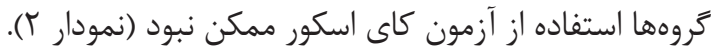

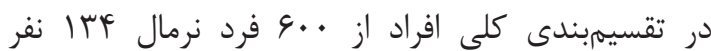

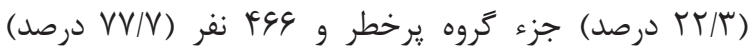

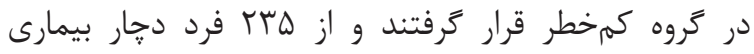

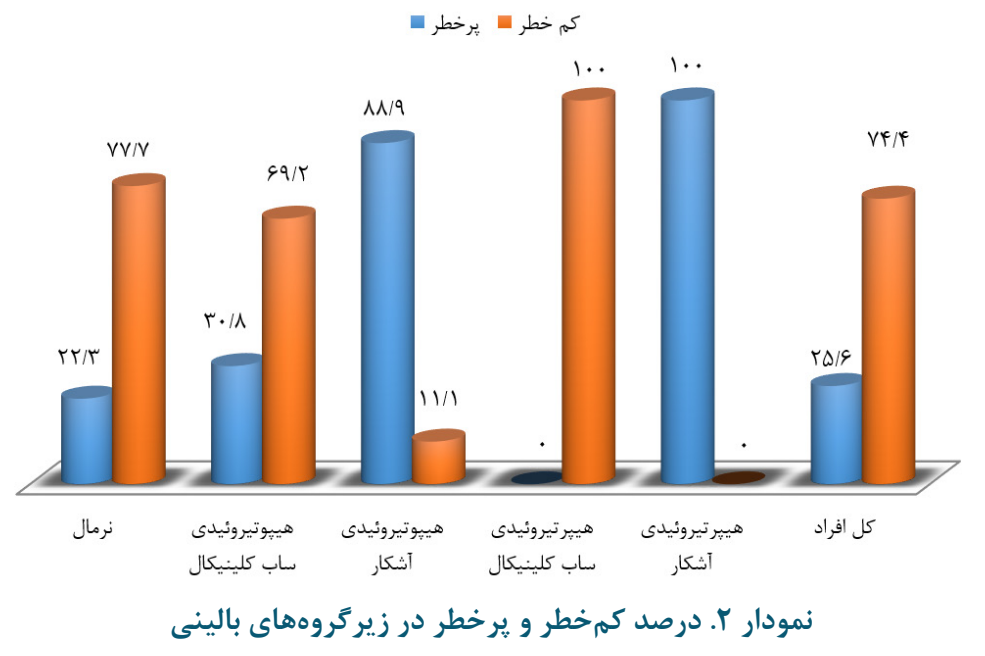

$\square \mathrm{TPO}+\square \mathrm{TPO}-$

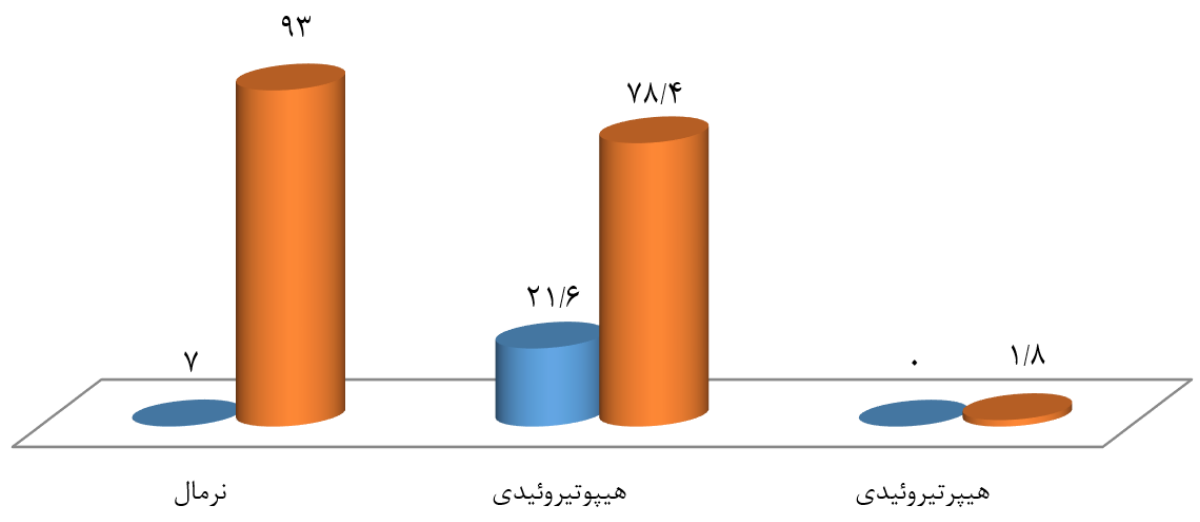

نمودار س. درصد آنتى TPO مثبت و منفى در افراد نرمال، هيير تيروئيدى، هييوتيروئيدى 


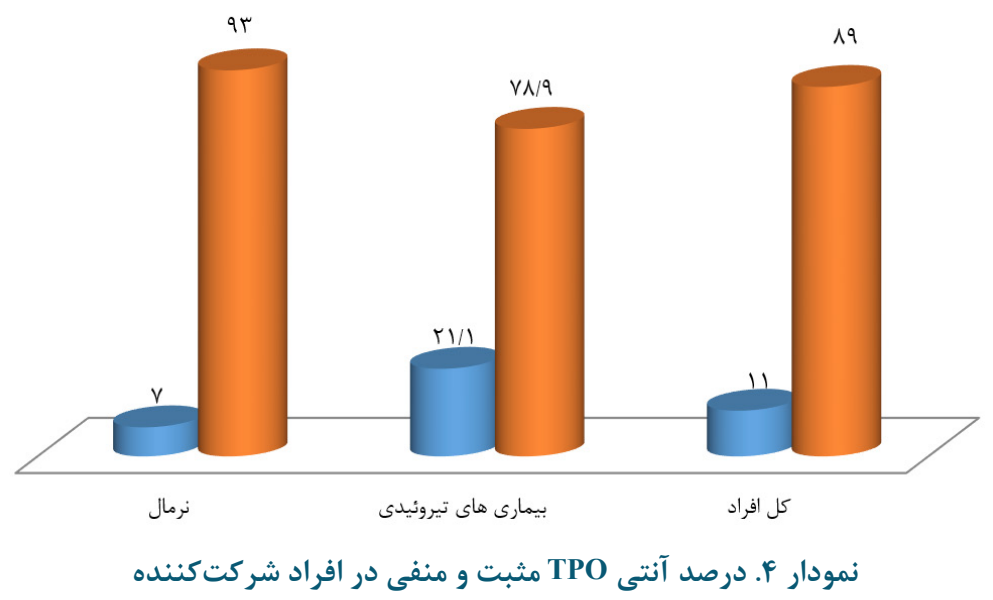

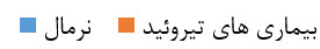

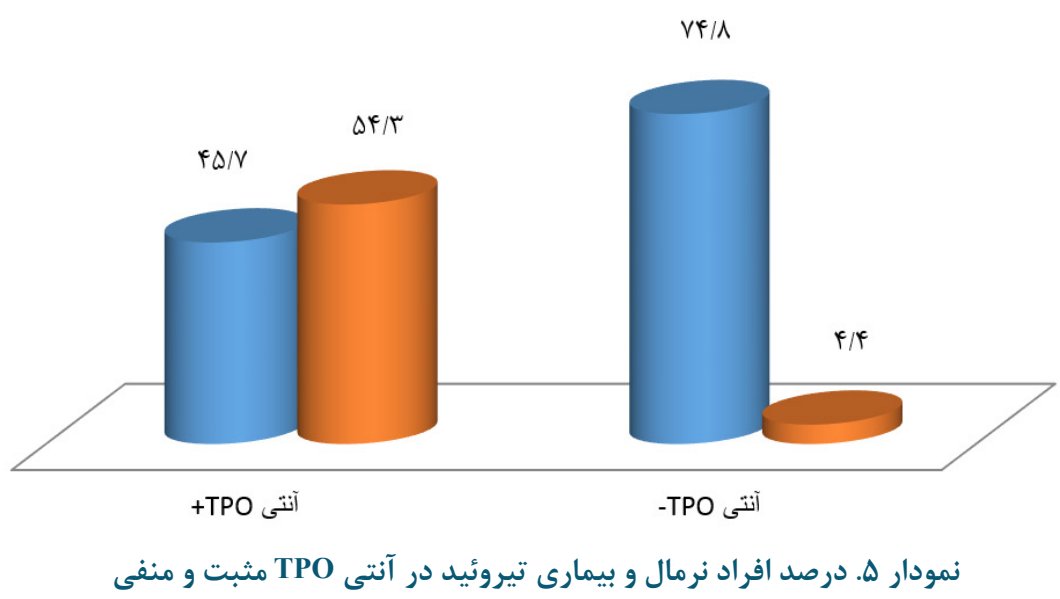

بالاى فرم تحت بالينى به يزوهشهاى ديخر درنظركرفتن

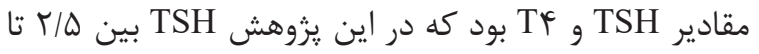

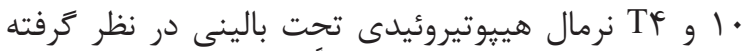

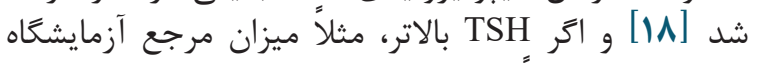

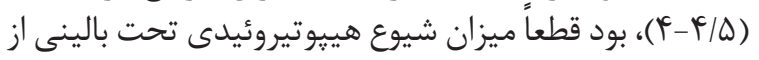

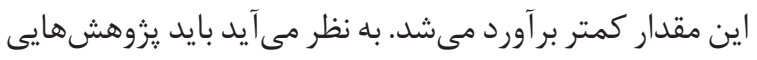

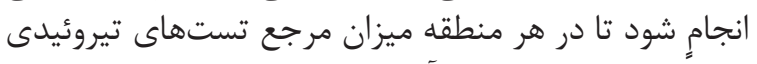

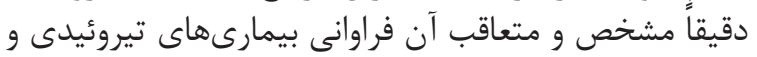
نيز موارد درمان آنها تعيين شود.

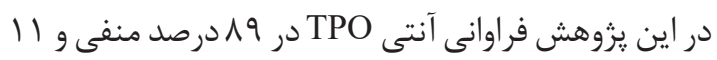

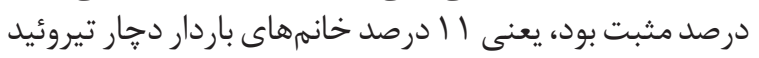

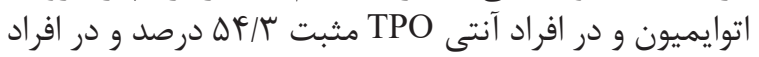

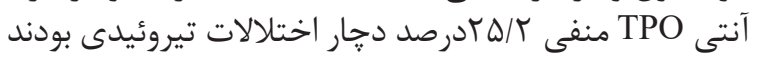

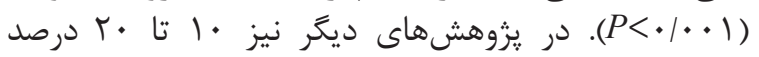

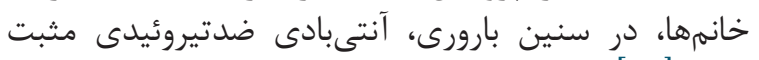

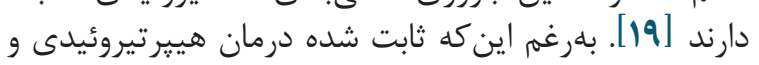

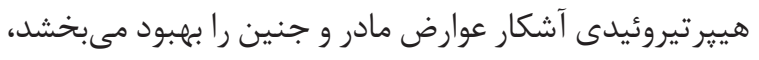

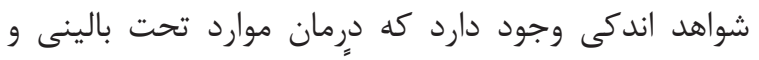

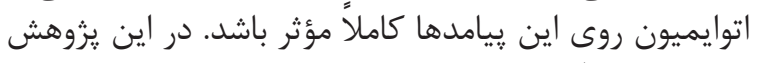

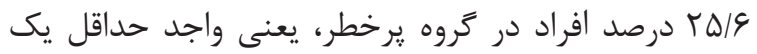

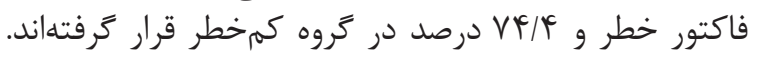

باردارى تأثيرات عمدهاى بر تنظيم عملكرد تيروئيد زنان

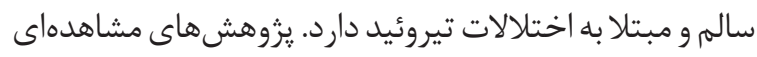

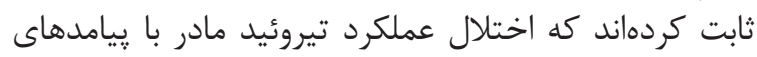

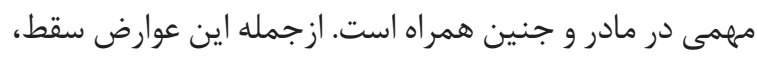

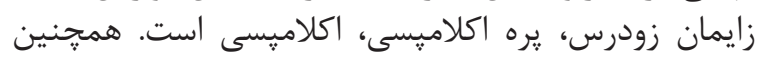

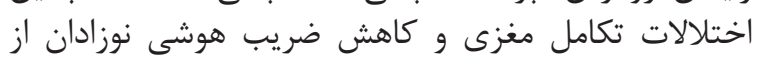

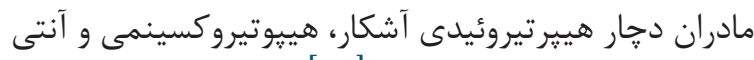
TPO مثبت كزارش شده است [IF

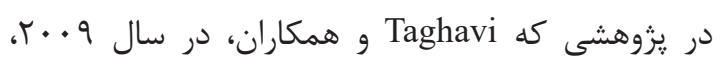

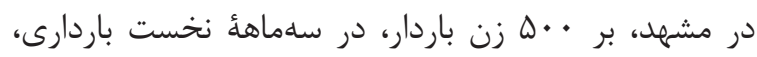

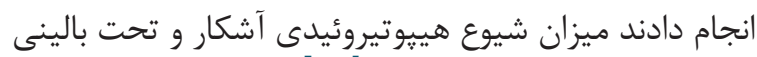

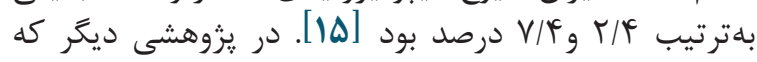
Naderi

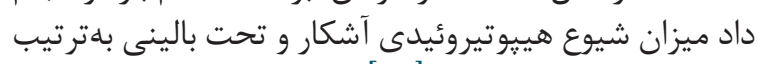

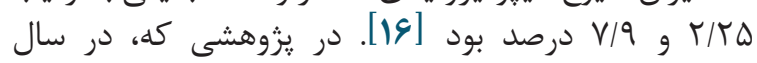
هير IrVV

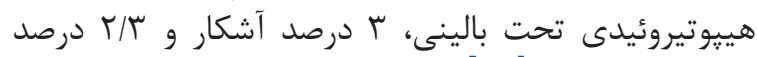

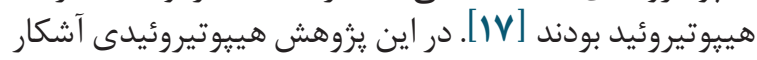

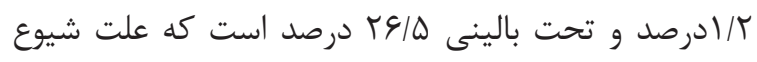

$$
\text { مجلئ مر اقبت يرستارى و مامايى ابنسينا }
$$




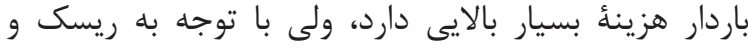

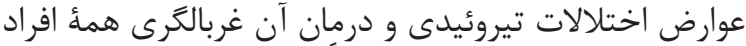

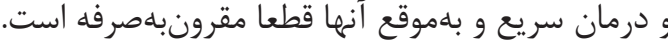

$$
\text { نتيجه }
$$

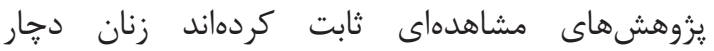

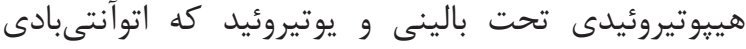

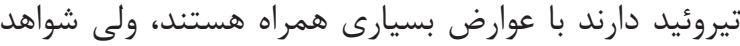

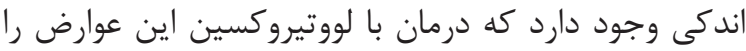

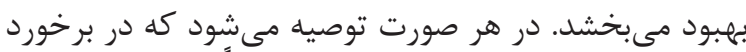
با يك خانم باردار در كلينيك شخصى درى حتماً

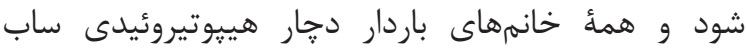

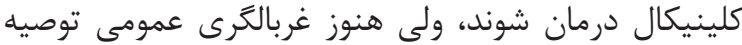

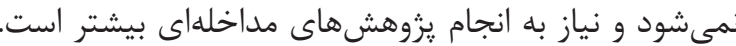

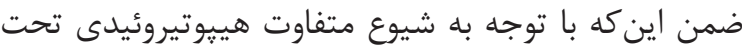

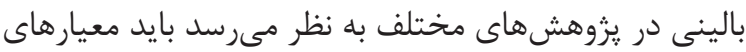

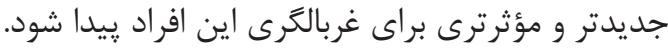

$$
\text { سباسگزارى }
$$

اين يزروهش با حمايت مالى دانشخاه علوم :زشكى همدان

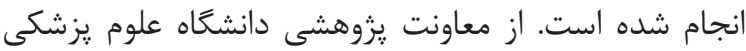

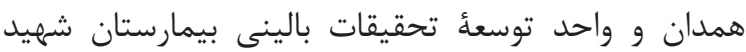

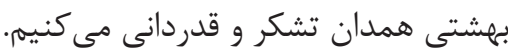

$$
\text { تعارض در منافع }
$$

بين نويسند

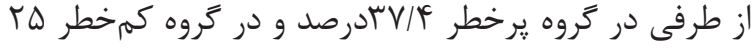

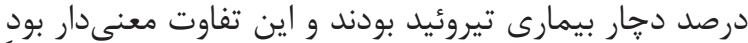

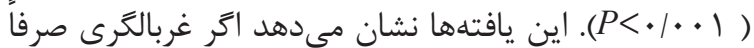

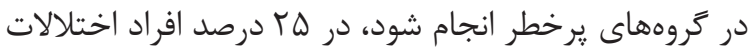

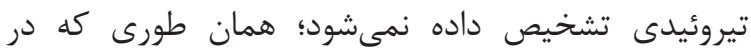

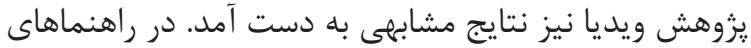

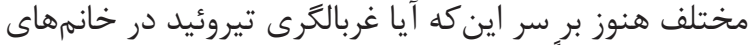

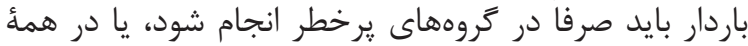

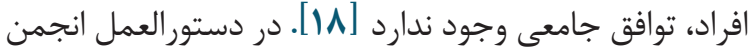

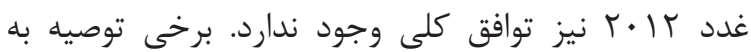

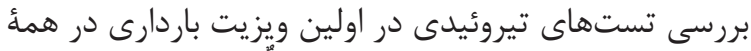

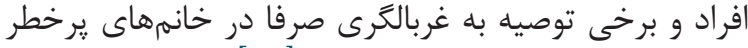

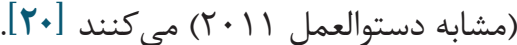

يزوهشهاى ديكرِى نيز وجود دارد كه بر بر اساس

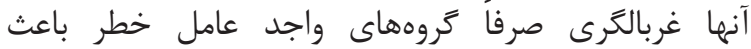

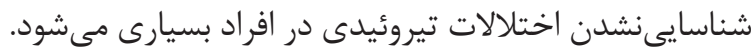

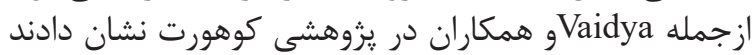
كه T/9 درصد خانمهاى باردار TSH بار

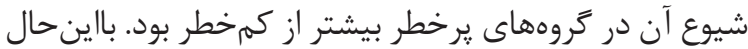

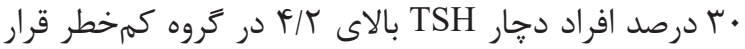

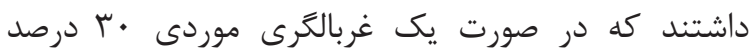

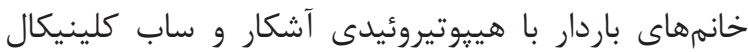
ناديده گرفته مىشدند [1/1].

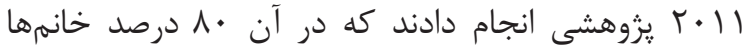

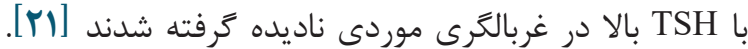

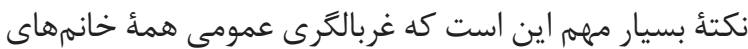

\section{References}

1. Van Raaij JA, Schonk C, Vermaat-Miedema S, Peek MM, Hautvast JA. Energy requirements of pregnancy in The Netherlands. The Lancet. 1987; 953-955. https://doi.org/10.1016/S01406736(87)91431-0

2. Glinoer D. The regulation of thyroid function in pregnancy: Pathways of endocrine adaptation from physiology to pathology. Endocrine Reviews. 1997; 18(3):404-433. https://doi. org/10.1210/edrv.18.3.0300

3. Alexander EK, Pearce EN, Brent GA, Brown RS, Chen H, Dosiou C, et al. 2017 Guidelines of the American Thyroid Association for the diagnosis and management of thyroid disease during pregnancy and the postpartum. Thyroid. 2017; 27(3):315-389. https://doi.org/10.1089/ thy. 2016.0457

4. Li C, Shan Z, Mao J, Wang W, Xie X, Zhou W, et al. Assessment of thyroid function during first-trimester pregnancy: What is the rational upper limit of serum TSH during the first trimester in Chinese pregnant women. The Journal of Clinical Endocrinology \& Metabolism. 2014; 99(1):73-9.

https://doi.org/10.1210/jc.2013-1674

5. Moon HW, Chung HJ, Park CM, Hur M, Yun Y-M. Establishment of trimester-specific reference intervals for thyroid hormones in Korean pregnant women. Annals of laboratory medicine. 2015; 35(2):198-204. https://doi.org/10.3343/ alm.2015.35.2.198

6. Glinoer D, Riahi M, Grün J, Kinthaert J. Risk of subclinical hypothyroidism in pregnant women with asymptomatic autoimmune thyroid disorders. The Journal of Clinical Endocrinology \& Metabolism. 1994; 79(1):197-204. https://doi. org/10.1210/jcem.79.1.8027226

7. Wang C. The relationship between type 2 diabetes mellitus and related thyroid diseases. Journal of diabetes research. 2013; 2013:390534. https:// doi.org/10.1155/2013/390534

8. Lahoti SK, Toppo L. Subclinical hypothyroidism and pregnancy outcomes. Annals of International Medical and Dental Research. 2015; 1(3):324326.

9. Korevaar TI, Schalekamp-Timmermans S, de 
Rijke YB, Visser WE, Visser W, de Muinck Keizer-Schrama SM, et al. Hypothyroxinemia and TPO-antibody positivity are risk factors for premature delivery: The generation R study. The Journal of Clinical Endocrinology \& Metabolism. 2013; 98(11):4382-4390. https://doi.org/10.1210/ jc. 2013-2855

10. Allan W, Haddow J, Palomaki G, Williams J, Mitchell M, Hermos R, et al. Maternal thyroid deficiency and pregnancy complications: implications for population screening. Journal of medical screening. 2000; 7(3):127-130. https://doi. org/10.1136/jms.7.3.127

11. Casey BM, Dashe JS, Spong CY, McIntire DD, Leveno KJ, Cunningham GF. Perinatal significance of isolated maternal hypothyroxinemia identified in the first half of pregnancy. Obstetrics \& Gynecology. 2007; 109(5):1129-1135. https:// doi.org/10.1097/01.AOG.0000262054.03531.24

12. Burch HB, Cooper DS. Management of Graves disease: A review. JAMA. 2015; 314(23):25442554. https://doi.org/10.1001/jama.2015.16535

13. Stagnaro-Green A, Abalovich M, Alexander E, Azizi F, Mestman J, Negro R, et al. Guidelines of the American Thyroid Association for the diagnosis and management of thyroid disease during pregnancy and postpartum. Thyroid. 2011; 21(10):1081-1125. https://doi.org/10.1089/ thy. 2011.0087

14. Pop VJ, de Vries E, van Baar AL, Waelkens J, De Rooy H, Horsten M, et al. Maternal thyroid peroxidase antibodies during pregnancy: a marker of impaired child development? The Journal of Clinical Endocrinology \& Metabolism. 1995; 80(12):3561-3566. $\quad$ https://doi.org/10.1210/ jcem.80.12.8530599

15. Taghavi M, Saghafi N, Shirin S. Outcome of thyroid dysfunction in pregnancy in Mashhad, Iran. International Journal of Endocrinology and Me- tabolism. 2009; 7(2):82-85.

16. Naderi T, Honarvar Z, Bahrampor A, Yosefzadeh GH. The Prevalence of Hypothyroidism Based on Risk Factors in Pregnant Women Referred to Shahid Dadbin Clinic, Kerman, Iran. Journal of Kerman University of Medical Sciences. 2012; 19(3):225-232.

17. Dehghani Zahedani M, Azinfar A, Mahouri K, Mehrdad S. The Identification of Related Risk Factors of Thyroid Disorder in an Iranian Pregnant Population. Iranian Journal of Endocrinology and Metabolism. 2010; 12(4):352-358

18. Vaidya B, Anthony S, Bilous M, Shields B, Drury J, Hutchison S, et al. Detection of thyroid dysfunction in early pregnancy: Universal screening or targeted high-risk case finding. The Journal of Clinical Endocrinology \& Metabolism. 2007; 92(1):203-207. https://doi.org/10.1210/jc.2006$\underline{1748}$

19. Thangaratinam S, Tan A, Knox E, Kilby MD, Franklyn J, Coomarasamy A. Association between thyroid autoantibodies and miscarriage and preterm birth: meta-analysis of evidence. BMJ. 2011; 342.

20. De Groot L, Abalovich M, Alexander EK, Amino $\mathrm{N}$, Barbour L, Cobin RH, et al. Management of thyroid dysfunction during pregnancy and postpartum: an Endocrine Society clinical practice guideline. The Journal of Clinical Endocrinology \& Metabolism. 2012; 97(8):2543-2565. https:// doi.org/10.1210/jc.2011-2803

21. Chang DL, Leung AM, Braverman LE, Pearce EN. Thyroid testing during pregnancy at an academic Boston Area Medical Center. The Journal of Clinical Endocrinology \& Metabolism. 2011; 96(9):E1452-E1456. https://doi.org/10.1210/ jc. 2011-0360 